\title{
Influence of Thermal Radiation on Unsteady MHD Free Convection Flow of Jeffrey Fluid over a Vertical Plate with Ramped Wall Temperature
}

\author{
Nor Athirah Mohd Zin, ${ }^{1}$ Ilyas Khan, ${ }^{2}$ and Sharidan Shafie ${ }^{1}$ \\ ${ }^{1}$ Department of Mathematical Sciences, Faculty of Science, Universiti Teknologi Malaysia, 81310 Johor Bahru, Malaysia \\ ${ }^{2}$ College of Engineering, Majmaah University, P.O. Box 66, Majmaah 11952, Saudi Arabia \\ Correspondence should be addressed to Ilyas Khan; i.said@mu.edu.sa
}

Received 20 September 2015; Revised 22 December 2015; Accepted 31 December 2015

Academic Editor: Mohsen Torabi

Copyright ( $) 2016$ Nor Athirah Mohd Zin et al. This is an open access article distributed under the Creative Commons Attribution License, which permits unrestricted use, distribution, and reproduction in any medium, provided the original work is properly cited.

Influence of thermal radiation on unsteady magnetohydrodynamic (MHD) free convection flow of Jeffrey fluid over a vertical plate with ramped wall temperature is studied. The Laplace transform technique is used to obtain the analytical solutions. Expressions for skin friction and Nusselt number are also obtained. Results of velocity and temperature distributions are shown graphically for embedded parameters such as Jeffrey fluid parameter $\lambda$, Prandtl number Pr, Grashof number Gr, Hartmann number Ha, radiation parameter $\mathrm{Rd}$, and dimensionless time $\tau$. It is observed that the amplitude of velocity and temperature profile for isothermal are always higher than ramped wall temperature.

\section{Introduction}

Investigation of non-Newtonian fluids has become very popular in this research area due to their wide range in industrial and technologies applications such as the plastic manufacture, performance of lubricants, food processing, or movement of biological fluids. Various models have been suggested to predict and describe the rheological behavior of non-Newtonian fluids. The Jeffrey fluid model is one of the subfamily of non-Newtonian fluids which has been given much attention in the present problem. This fluid is a relatively simple viscoelastic non-Newtonian fluid model that exhibits both relaxation and retardation effects [1].

Hayat and Mustafa [2] discussed the effect of the thermal radiation on the unsteady mixed convection flow of Jeffrey fluid past a porous vertical stretching surface analytically using homotopy analysis method. Hayat et al. [3] extended the previous idea to examine the flow of an incompressible Jeffrey fluid over a stretching surface in the presence of power heat flux and heat source. Shehzad et al. [4] obtained homotopy solutions for magnetohydrodynamic radiative flow of an incompressible Jeffrey fluid over a linearly stretched surface. In another investigation, Shehzad et al. [5] discussed the three-dimensional hydromagnetic flow of Jeffrey fluid with nanoparticles where the effects of thermal radiation and internal heat generation are considered. Hussain et al. [6] conducted heat and mass transfer analysis of twodimensional hydromagnetic flow of an incompressible Jeffrey nanofluid over an exponentially stretching surface in the presence of thermal radiation, viscous dissipation, Brownian motion, and thermophoresis effects. Very recently, the influence of melting heat transfer and thermal radiation on MHD stagnation point flow of an electrically conducting Jeffrey fluid over a stretching sheet with partial surface slip has been analyzed numerically by Das et al. [7] with the help of RungeKutta-Fehlberg method.

Besides that, Jeffrey fluid also has been studied in peristalsis known as major mechanism of fluid transport especially in biological system, for example, urine transport from kidney to bladder through ureter, movement of chime in the gastrointestinal tract, the movement of spermatozoa in the ducts efferent of the male reproductive tract, ovum in the female fallopian tube, the locomotion 
of some worms, and transport of lymph in the lymphatic vessels [8]. Abd-Alla et al. [9] investigated magnetic field and gravity effects on peristaltic transport of a Jeffrey fluid in an asymmetric channel. Quite recently, Abd-Alla and Abo-Dahab [10] analyzed the influence of magnetic field and rotation effects on peristaltic transport of a Jeffrey fluid in asymmetric channel. Akram and Nadeem [11] provided exact and closed form Adomian solutions for the peristaltic motion of two-dimensional Jeffrey fluid in an asymmetric channel under the effects of induced magnetic field and heat transfer.

Moreover, the influence of heat transfer on MHD oscillatory flow of Jeffrey fluid in a channel has been investigated by Kavita et al. [12]. Idowu et al. [13] studied the effects of heat and mass transfer on unsteady MHD oscillatory flow of Jeffrey fluid in a horizontal channel with chemical reaction and solved analytically using perturbation technique. Ali and Asghar [14] presented analytical solution for twodimensional oscillatory flow inside a rectangular channel for Jeffrey fluid with small suction. They used perturbation, Wentzel-Kramers-Brillouin, and variation of parameter as the methodologies to solve this complex problem. Detailed discussions on oscillatory flow of Jeffrey fluid also can be found in Khan [15] and Hayat et al. [1, 16].

On the other hand, the steady boundary layer flow of a Jeffrey fluid over a shrinking sheet is highlighted by Nadeem et al. [17]. Hamad et al. [18] presented a study of thermal jump effects on boundary layer flow of a Jeffrey fluid near the stagnation point on a stretching/shrinking sheet with variable thermal conductivity and solved numerically using finite difference method. Hayat et al. [19] considered twodimensional stagnation point flow of Jeffrey fluid over a convectively heated stretching sheet constructed by homotopy analysis method. Other than that, Qasim [20] observed the combined effect of heat and mass transfer in Jeffrey fluid over a stretching sheet in the presence of heat source/heat sink where the obtained exact solutions are derived by power series method using Kummer's confluent hypergeometric functions. Dalir [21] investigated entropy generation for the steady two-dimensional laminar forced convection flow and heat transfer of an incompressible Jeffrey non-Newtonian fluid over a linearly stretching, impermeable, and isothermal sheet numerically by Keller's box method.

Diverse contributions dealing with boundary layer flow over stretching sheet in various situations are well documented in Dalir et al. [22] and Hayat et al. [23]. Hayat et al. [24] analyzed the effects of Newtonian heating magnetohydrodynamics (MHD) in a flow of a Jeffrey fluid over a radially stretching surface. In the same year, Hayat et al. [25] carried out the study of boundary layer stretched flow of a Jeffrey fluid subject to the convective boundary condition. Shehzad et al. [26] analytically discussed magnetohydrodynamics (MHD) three-dimensional flow of Jeffrey fluid in the presence of Newtonian heating. Farooq et al. [27] examined the combined effects of Joule and Newtonian heating in magnetohydrodynamic (MHD) flow of Jeffrey fluid over a stretching cylinder with heat source/sink solved analytically by homotopy analysis method (HAM), whereas Khan [28] recently implemented the Laplace transform technique to find the exact solutions for unsteady free convection flow of a Jeffrey fluid past an infinite isothermal vertical plate.

In view of the above analysis, so far no study has been made to examine the effect of ramped wall temperature in a Jeffrey fluid flow. Thus, our main focus is to discuss the influence of thermal radiation on unsteady MHD free convection flow of Jeffrey fluid over a vertical plate with ramped wall temperature. The Laplace transform technique is applied in this study in order to find the analytical solutions for velocity and temperature profiles. Expressions of skin friction and Nusselt number are also given. Graphical results are provided and discussed for embedded parameters.

\section{Mathematical Formulation of the Problem}

The unsteady incompressible flow is described by the following governing equations:

$$
\begin{aligned}
\nabla \cdot \mathbf{V} & =0, \\
\rho\left[\frac{\partial \mathbf{V}}{\partial t}+(\mathbf{V} \cdot \nabla) \mathbf{V}\right] & =\operatorname{div} \mathbf{T}+\mathbf{J} \times \mathbf{B}+\rho \mathbf{g}, \\
\operatorname{curl} \mathbf{E} & =-\frac{\partial \mathbf{B}}{\partial t}, \\
\operatorname{curl} \mathbf{B} & =\mu_{m} \mathbf{J}, \\
\nabla \cdot \mathbf{B} & =0,
\end{aligned}
$$

In the above equations $\mathbf{V}$ indicates the velocity field, $\mathbf{T}$ is the Cauchy stress tensor, $\rho$ is the fluid density, $\mathbf{J}$ is the current density, $\mathbf{B}=B_{0}+b$ is the total magnetic field, where $B_{0}$ and $b$ are the applied and induced magnetic fields, respectively, $\mathbf{g}$ is the gravitational force which is downward in $x$-direction, $\mathbf{E}$ is the total electric field current, and $\mu_{m}$ is the magnetic permeability. The constitutive equations for Jeffrey fluid are given by

$$
\begin{aligned}
& \mathbf{T}=-p \mathbf{I}+\mathbf{S} \\
& \mathbf{S}=\frac{\mu}{1+\lambda_{1}}\left[\mathbf{A}_{1}+\lambda_{2}\left(\frac{\partial \mathbf{A}_{1}}{\partial t}+(\mathbf{V} \cdot \nabla) \mathbf{A}_{1}\right)\right]
\end{aligned}
$$

in which $-p \mathbf{I}$ is the indeterminate part of the stress, $\mathbf{S}$ is the extra tensor, $\mu$ is the dynamic viscosity, and $\lambda_{1}$ and $\lambda_{2}$ are the material parameters of the Jeffrey fluid known as the ratio of relaxation to retardation times and retardation time, respectively. Notice that if $\lambda_{1}=\lambda_{2}=0$, it will lead to the expression of an incompressible viscous fluid. The RivlinEricksen tensor, $\mathbf{A}_{1}$, is defined by

$$
\mathbf{A}_{1}=\nabla \mathbf{V}+(\nabla \mathbf{V})^{T} .
$$

For unidirectional flow, we assume that velocity and stress fields are in the form of

$$
\begin{aligned}
& \mathbf{V}=\mathbf{V}(y, t)=u(y, t) \mathbf{i}, \\
& \mathbf{S}=\mathbf{S}(y, t),
\end{aligned}
$$

where $u$ is the $x$-component of velocity $\mathbf{V}$ and $\mathbf{i}$ is the unit vector along the $x$-direction of the Cartesian coordinate 


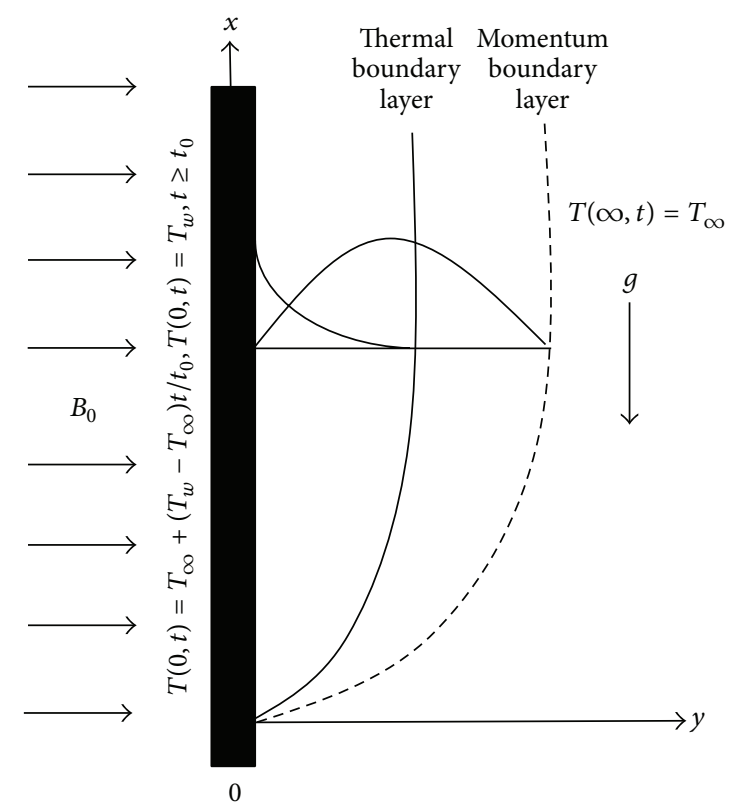

FIgURE 1: Schematic diagram for the flow of Jeffrey fluid past a vertical plate.

system. The stress field satisfies the condition $\mathbf{S}(y, 0)=0$ which gives the following results:

$$
\begin{aligned}
& S_{x x}=S_{y y}=S_{z z}=S_{x z}=S_{z x}=S_{y z}=S_{z y}=0, \\
& S_{x y}=\frac{\mu}{1+\lambda_{1}}\left(1+\lambda_{2} \frac{\partial}{\partial t}\right) \frac{\partial u}{\partial y},
\end{aligned}
$$

where $S_{x y}$ is the nontrivial tangential stress.

Consider the effect of thermal radiation on unsteady free convection flow of Jeffrey fluid near the vertical plate situated in the $(x, z)$ plane of a Cartesian coordinate system of $x, y$, and $z$ as presented in Figure 1. It is assumed that, at time $t \leq 0$, both plate and fluid are at rest at constant temperature $T_{\infty}$. At time $t>0$, the temperature of the plate is raised or lowered to $T_{\infty}+\left(T_{w}-T_{\infty}\right)\left(t / t_{0}\right)$ when $t \leq t_{0}$, and the constant temperature $T_{w}$ is maintained at $t>t_{0}$.

Under the above assumption and applying the usual Boussinesq approximation, the momentum and energy equations for unsteady free convection flow of Jeffrey fluid past an infinite vertical plate are given by

$$
\begin{aligned}
\rho \frac{\partial u}{\partial t}= & \frac{\mu}{1+\lambda_{1}}\left(1+\lambda_{2} \frac{\partial}{\partial t}\right) \frac{\partial^{2} u}{\partial y^{2}}+\rho g \beta\left(T-T_{\infty}\right) \\
& -\sigma B_{0}^{2} u \\
\rho c_{p} \frac{\partial T}{\partial t}= & k \frac{\partial^{2} T}{\partial y^{2}}-\frac{\partial q_{r}}{\partial y},
\end{aligned}
$$

with appropriate initial and boundary conditions

$$
\begin{aligned}
& u(y, 0)=0, \\
& T(y, 0)=T_{\infty}, \\
& y>0, \\
& u(0, t)=0, \quad t>0, \\
& T(0, t)=T_{\infty}+\left(T_{w}-T_{\infty}\right) \frac{t}{t_{0}}, \quad 0<t<t_{0}, \\
& T(0, t)=T_{w}, \quad t \geq t_{0}, \\
& u(\infty, t)=0, \\
& T(\infty, t)=T_{\infty},
\end{aligned}
$$

where $u$ is the fluid velocity in $x$-direction, $T$ is the temperature, $\rho$ is the constant density of the fluid, $g$ is the acceleration due to gravity, $c_{p}$ is the specific heat capacity, $k$ is the thermal conductivity, $T_{w}$ is the wall temperature, $T_{\infty}$ is the free stream temperature, and $q_{r}$ is the radiation heat flux, respectively. Using Rosseland approximation, the radiation heat flux can be written as

$$
q_{r}=-\frac{4 \sigma^{*}}{3 k_{1}} \frac{\partial T^{4}}{\partial y}
$$

where $\sigma^{*}$ denotes the Stefan-Boltzmann and $k_{1}$ is the absorption coefficient. We assume that the temperature differences within the flow are sufficiently small such that $T_{4}$ can be expanded in Taylor series. Hence, expanding $T_{4}$ about $T_{\infty}$ and neglecting higher order terms, we get $T^{4} \approx 4 T_{\infty}^{3} T-3 T_{\infty}^{4}$. Therefore, (7) is simplified to

$$
\rho c_{p} \frac{\partial T}{\partial t}=k\left(1+\frac{16 \sigma^{*} T_{\infty}^{3}}{3 k k_{1}}\right) \frac{\partial^{2} T}{\partial y^{2}} .
$$

Introducing the dimensionless variables

$$
\begin{aligned}
u^{*} & =\frac{u}{U_{0}}, \\
\eta^{*} & =\frac{y U_{0}}{v}, \\
\tau^{*} & =\frac{t U_{0}^{2}}{v}, \\
\theta & =\frac{T-T_{\infty}}{T_{w}-T_{\infty}}
\end{aligned}
$$

into (6) and (10) yields the following dimensionless equations (* notations are dropped for simplicity):

$$
\begin{aligned}
\frac{\partial u}{\partial \tau} & =\frac{1}{1+\lambda_{1}}\left(1+\lambda \frac{\partial}{\partial \tau}\right) \frac{\partial^{2} u}{\partial \eta^{2}}-\operatorname{Ha} u+\operatorname{Gr} \theta, \\
\operatorname{Pr} \frac{\partial \theta}{\partial \tau} & =(1+\mathrm{Rd}) \frac{\partial^{2} \theta}{\partial \eta^{2}},
\end{aligned}
$$


where $\lambda$ is the dimensionless Jeffrey fluid parameter, $\mathrm{Ha}$ is the Hartmann number, $\mathrm{Gr}$ is the Grashof number, $\mathrm{Pr}$ is the Prandtl number, and Rd is the radiation parameter which are denoted as follows:

$$
\begin{aligned}
\lambda & =\frac{\lambda_{2} U_{0}^{2}}{v}, \\
\mathrm{Ha} & =\frac{\sigma B_{0}^{2} v}{\rho U_{0}^{2}}, \\
\mathrm{Gr} & =\frac{g \beta v\left(T_{w}-T_{\infty}\right)}{U_{0}^{3}}, \\
\operatorname{Pr} & =\frac{\mu c_{p}}{k}, \\
\mathrm{Rd} & =\frac{16 \sigma^{*} T_{\infty}^{3}}{3 k k_{1}} .
\end{aligned}
$$

Also, initial and boundary conditions (8) become

$$
\begin{gathered}
u(\eta, 0)=0, \\
\theta(\eta, 0)=0, \\
\eta>0, \\
u(0, \tau)=0, \quad \tau>0, \\
\theta(0, \tau)=\tau, \quad 0<\tau \leq 1, \\
\theta(0, \tau)=1, \quad \tau>1, \\
u(\infty, \tau)=0, \quad \\
\theta(\infty, \tau)=0, \\
\tau>0 .
\end{gathered}
$$

\section{Solution of the Problem}

Applying the Laplace transform to (12) and (14) yields the following equations in the transformed $(\eta, q)$-plane:

$$
\begin{aligned}
& \frac{d^{2} \bar{u}(\eta, q)}{d \eta^{2}}-\left(\frac{\left(1+\lambda_{1}\right)(\mathrm{Ha}+q)}{1+\lambda q}\right) \bar{u}(\eta, q) \\
& =-\operatorname{Gr}\left(\frac{\left(1+\lambda_{1}\right)}{1+\lambda q}\right) \bar{\theta}(\eta, q), \\
& (1+\mathrm{Rd}) \frac{d^{2} \bar{\theta}(\eta, q)}{d \eta^{2}}-\operatorname{Pr} q \bar{\theta}(\eta, q)=0,
\end{aligned}
$$

and initial and boundary conditions are transformed as follows:

$$
\begin{aligned}
\bar{u}(0, q) & =0, \\
\bar{\theta}(0, q) & =\frac{1-\exp (-q)}{q^{2}}, \\
\bar{u}(\infty, q) & =0, \\
\bar{\theta}(\infty, q) & =0,
\end{aligned}
$$

where $\bar{u}(\eta, q)$ and $\bar{\theta}(\eta, q)$ are Laplace transforms of $u(\eta, \tau)$ and $\theta(\eta, \tau)$.

Equation (16) subject to boundary conditions (17) has the following solution:

$$
\bar{\theta}(\eta, q)=\frac{1-\exp (-q)}{q^{2}} \exp \left(-\eta \sqrt{\frac{\operatorname{Pr} q}{1+\mathrm{Rd}}}\right) .
$$

Denote

$$
\begin{aligned}
\theta_{1}(\eta, \tau)= & L^{-1}\left\{\frac{1}{q^{2}} \exp \left(-\eta \sqrt{\frac{\operatorname{Pr}}{1+\mathrm{Rd}}} \sqrt{q}\right)\right\} \\
= & \left(\frac{\eta^{2}}{2} \frac{\operatorname{Pr}}{1+\mathrm{Rd}}+\tau\right) \operatorname{erfc}\left(\frac{\eta}{2 \sqrt{\tau}} \sqrt{\frac{\operatorname{Pr}}{1+\mathrm{Rd}}}\right) \\
& -\frac{\eta}{\sqrt{\pi}} \sqrt{\frac{\operatorname{Pr} \tau}{1+\mathrm{Rd}}} \exp \left(-\frac{\eta^{2}}{4 \tau} \frac{\operatorname{Pr}}{1+\mathrm{Rd}}\right) .
\end{aligned}
$$

And using the second shift property, we obtain the following solution for temperature distribution:

$$
\theta(\eta, \tau)=\theta_{1}(\eta, \tau)-\theta_{1}(\eta, \tau-1) H(\tau-1),
$$

where $H(\cdot)$ is Heaviside function and $\operatorname{erfc}(\cdot)$ denote the complementary error function.

Meanwhile, the solution of (15) under boundary conditions (17) results in

$$
\bar{u}(\eta, q)=[1-\exp (-q)] \bar{u}_{3}(q) \cdot \bar{u}_{4}(\eta, q),
$$

where

$$
\begin{aligned}
& \bar{u}_{3}(q)=\frac{\operatorname{Gr}(1+\operatorname{Rd})\left(1+\lambda_{1}\right)}{\operatorname{Pr} \lambda} \frac{1}{\left(q+m_{1}\right)^{2}-m_{2}^{2}}, \\
& \bar{u}_{4}(\eta, q)=\frac{1}{q^{2}}\left\{\exp \left(-\eta \sqrt{\frac{\left(1+\lambda_{1}\right)(\mathrm{Ha}+q)}{1+\lambda q}}\right)\right. \\
& \left.-\exp \left(-\eta \sqrt{\frac{\operatorname{Pr} q}{1+\mathrm{Rd}}}\right)\right\},
\end{aligned}
$$


with

$$
\begin{aligned}
& m_{1}=\frac{\operatorname{Pr}-\left(1+\lambda_{1}\right)(1+\mathrm{Rd})}{2 \operatorname{Pr} \lambda}, \\
& m_{2}=\sqrt{\frac{\left(\left(\operatorname{Pr}-\left(1+\lambda_{1}\right)(1+\mathrm{Rd})\right) / \operatorname{Pr} \lambda\right)^{2}+4\left(1+\lambda_{1}\right)(1+\mathrm{Rd}) \mathrm{Ha} / \operatorname{Pr} \lambda}{4}} .
\end{aligned}
$$

The inverse Laplace transform of (22) leads to the following expressions:

$$
\begin{aligned}
u_{3}(\tau) & =L^{-1}\left\{\bar{u}_{3}(q)\right\} \\
& =\frac{\operatorname{Gr}(1+\mathrm{Rd})\left(1+\lambda_{1}\right)}{\operatorname{Pr} \lambda m_{2}} e^{-m_{1} \tau} \sinh \left(m_{2} \tau\right) .
\end{aligned}
$$

In order to determine the inverse Laplace transform of $u_{4}(\eta, \tau)$ of the function $\bar{u}_{4}(\eta, q)$, we split (23) in the following forms:

$$
\begin{aligned}
& \bar{A}_{1}(\eta, q)=\frac{1}{q^{2}} \exp \left(-\eta \sqrt{\frac{\left(1+\lambda_{1}\right)(\mathrm{Ha}+q)}{1+\lambda q}}\right), \\
& \bar{A}_{2}(\eta, q)=\frac{1}{q^{2}} \exp \left(-\eta \sqrt{\frac{\operatorname{Pr} q}{1+\mathrm{Rd}}}\right) .
\end{aligned}
$$

Using the inversion formula of compound function and the inverse Laplace formula into (26) implies

$$
\begin{aligned}
& A_{1}(\eta, \tau)=L^{-1}\left\{\bar{A}_{1}(\eta, q)\right\}=\tau e^{-\eta / \sqrt{k_{1}}}+\frac{\eta}{2 k_{1}} \\
& \cdot \sqrt{\frac{b}{\pi}} \int_{0}^{\tau} \int_{0}^{\infty} \frac{(\tau-s)}{u \sqrt{u}} \sqrt{\frac{u}{s}} e^{-\left(\left(k_{2} s+u\right) / k_{1}+\eta^{2} / 4 u\right)} I_{1}\left(\frac{2}{k_{2}}\right. \\
& \cdot \sqrt{b u s}) d u d s,
\end{aligned}
$$

and the inverse Laplace transform of (27) is given by

$$
\begin{aligned}
A_{2}(\eta, \tau)= & L^{-1}\left\{\bar{A}_{2}(\eta, q)\right\} \\
= & \left(\tau+\frac{\eta^{2}}{2} \frac{\operatorname{Pr}}{1+\mathrm{Rd}}\right) \operatorname{erfc}\left(\frac{\eta}{2} \sqrt{\frac{\operatorname{Pr}}{\tau(1+\mathrm{Rd})}}\right) \\
& -\eta \sqrt{\frac{\operatorname{Pr} \tau}{\pi(1+\mathrm{Rd})}} e^{-\left(\left(\eta^{2} / 4 \tau\right)(\operatorname{Pr} /(1+\mathrm{Rd}))\right)} .
\end{aligned}
$$

$$
\begin{aligned}
& u_{R 1}(\eta, \tau)=\frac{\mathrm{Gr}(1+\mathrm{Rd})\left(1+\lambda_{1}\right)}{\operatorname{Pr} \lambda m_{2}} \int_{0}^{\tau} p e^{-\left(m_{1}(\tau-p)+\eta / \sqrt{k_{1}}\right)} \sinh \left(m_{2}(\tau-p)\right) d p+\frac{\mathrm{Gr}(1+\mathrm{Rd})\left(1+\lambda_{1}\right)}{2 \lambda k_{1} m_{2} \operatorname{Pr}} \\
& \cdot \sqrt{\frac{b}{\pi}} \int_{0}^{\tau} \int_{0}^{p} \int_{0}^{\infty}\left\{\frac{\eta(p-s)}{u \sqrt{s}} e^{-\left(m_{1}(\tau-p)+\left(k_{2} s+u\right) / k_{1}+\eta^{2} / 4 u\right)} \sinh \left(m_{2}(\tau-p)\right) I_{1}\left(\frac{2}{k_{1}} \sqrt{b u s}\right)\right\} d u d s d p
\end{aligned}
$$

Thus,

$$
\begin{aligned}
& u_{4}(\eta, \tau)=\tau e^{-\eta / \sqrt{k_{1}}}+\frac{\eta}{2 k_{1}} \\
& \cdot \sqrt{\frac{b}{\pi} \int_{0}^{\tau} \int_{0}^{\infty} \frac{(\tau-s)}{u \sqrt{u}} \sqrt{\frac{u}{s}} e^{-\left(\left(k_{2} s+u\right) / k_{1}+\eta^{2} / 4 u\right)} I_{1}\left(\frac{2}{k_{2}}\right.} \\
& \cdot \sqrt{b u s}) d u d s-\left(\tau+\frac{\eta^{2}}{2} \frac{\operatorname{Pr}}{1+\mathrm{Rd}}\right) \\
& \cdot \operatorname{erfc}\left(\frac{\eta}{\frac{\operatorname{Pr}}{\tau(1+\mathrm{Rd})}}\right) \\
& +\eta \sqrt{\frac{\operatorname{Pr} \tau}{\pi(1+\mathrm{Rd})} e^{-\left(\left(\eta^{2} / 4 \tau\right)(\operatorname{Pr} /(1+\mathrm{Rd}))\right)}}
\end{aligned}
$$

where $k_{1}=\lambda /\left(1+\lambda_{1}\right), k_{2}=1 /\left(1+\lambda_{1}\right), b=k_{2}-k_{1} \mathrm{Ha}$, and $I_{1}(\cdot)$ is modified Bessel function of the first kind of order one. Consequently, the expression for velocity distribution can be written as

$$
u(\eta, \tau)=u_{R 1}(\eta, \tau)-u_{R 1}(\eta, \tau-1) H(\tau-1),
$$

where $u_{R 1}(\eta, \tau)$ can be found by using convolution theorem

$$
\begin{aligned}
u_{R 1}(\eta, \tau) & =\left(u_{3} * u_{4}\right)(\tau) \\
& =\int_{0}^{\tau} u_{3}(\tau-p) u_{4}(\eta, p) d p
\end{aligned}
$$

in which 


$$
\begin{aligned}
& -\frac{\mathrm{Gr}(1+\mathrm{Rd})\left(1+\lambda_{1}\right)}{\operatorname{Pr} \lambda m_{2}} \int_{0}^{\tau}\left\{e ^ { - m _ { 1 } ( \tau - p ) } ( p + \frac { \eta ^ { 2 } } { 2 } \frac { \mathrm { Pr } } { 1 + \mathrm { Rd } } ) \operatorname { s i n h } ( m _ { 2 } ( \tau - p ) ) \operatorname { e r f c } \left(\frac{\eta}{2 \sqrt{p}} \sqrt{\left.\left.\frac{\operatorname{Pr}}{1+\mathrm{Rd}}\right)\right\} d p}\right.\right. \\
& +\frac{\mathrm{Gr}\left(1+\lambda_{1}\right)}{\lambda m_{2}} \sqrt{\frac{1+\mathrm{Rd}}{\pi \operatorname{Pr}}} \int_{0}^{\tau}\left\{\eta \sqrt{p} e^{-\left(m_{1}(\tau-p)+\left(\eta^{2} / 4 p\right)(\operatorname{Pr} /(1+\mathrm{Rd}))\right)} \sinh \left(m_{2}(\tau-p)\right)\right\} d p .
\end{aligned}
$$

\section{Solution for an Isothermal Plate}

The analytical solution of temperature and velocity distribution for the case of ramped wall temperature are shown in (20) and (31). It is good to compare these results with those corresponding to the flow near a plate with uniform temperature to see the effects of ramped wall temperature of the plate on the fluid flow. The solution for temperature profile can be obtained as

$$
\theta(\eta, \tau)=\operatorname{erfc}\left(\frac{\eta}{2 \sqrt{\tau}} \sqrt{\frac{\mathrm{Pr}}{1+\mathrm{Rd}}}\right),
$$

and using the same way as before, the expression of velocity profile for case of isothermal is

$$
\begin{aligned}
& u(\eta, \tau)=\frac{\operatorname{Gr}(1+\mathrm{Rd})\left(1+\lambda_{1}\right)}{\operatorname{Pr} \lambda m_{2}} \int_{0}^{\tau} \exp \left(-m_{1}(\tau-p)-\frac{\eta}{\sqrt{k_{1}}}\right) \sinh \left(m_{2}(\tau-p)\right) d p+\frac{\operatorname{Gr}(1+\operatorname{Rd})\left(1+\lambda_{1}\right)}{\operatorname{Pr} \lambda m_{2}} \\
& \cdot \int_{0}^{\tau} \int_{0}^{p} \int_{0}^{\infty}\left\{\frac{\eta}{2 u \sqrt{\pi u}} \sqrt{\frac{b u}{s}} \frac{1}{k_{1}} \exp \left[-\left(m_{1}(\tau-p)+\frac{k_{2} s+u}{k_{1}}+\frac{\eta^{2}}{4 u}\right)\right] \sinh \left(m_{2}(\tau-p)\right) I_{1}\left(\frac{2}{k_{1}} \sqrt{b u s}\right)\right\} d u d s d p \\
& -\frac{\operatorname{Gr}(1+\operatorname{Rd})\left(1+\lambda_{1}\right)}{\operatorname{Pr} \lambda m_{2}} \int_{0}^{\tau} \exp \left(-m_{1}(\tau-p)\right) \sinh \left(m_{2}(\tau-p)\right) \operatorname{erfc}\left(\frac{\eta}{2 \sqrt{p}} \sqrt{\frac{\operatorname{Pr}}{1+\mathrm{Rd}}}\right) d p .
\end{aligned}
$$

\section{Nusselt Number and Skin Friction}

The expression of Nusselt number, $\mathrm{Nu}$, and skin friction, $F(\tau)$, for both cases, ramped wall temperature and isothermal, is discussed in this section. The Nusselt number and skin friction for ramped wall temperature can be written as

$$
\mathrm{Nu}=-\left.\frac{\partial \theta}{\partial \eta}\right|_{\eta=0}
$$

$$
\begin{aligned}
& =\frac{2}{\sqrt{\pi}} \sqrt{\frac{\operatorname{Pr}}{1+\mathrm{Rd}}}[\sqrt{\tau}-\sqrt{\tau-1} H(\tau-1)], \\
F(\eta, \tau) & =\left.\frac{1}{1+\lambda_{1}}\left(1+\lambda \frac{\partial}{\partial \tau}\right) \frac{\partial u}{\partial \eta}\right|_{\eta=0} \\
& =F_{1}(\tau)-F_{1}(\tau-1) H(\tau-1),
\end{aligned}
$$

where

$$
\begin{aligned}
& F_{1}(\tau)=-\frac{\operatorname{Gr}(1+\mathrm{Rd})}{\operatorname{Pr} \sqrt{k_{1}}} \int_{0}^{\tau}\left\{p e^{-m_{1}(\tau-p)}\left[\frac{\left(1-\lambda m_{1}\right)}{\lambda m_{2}} \sinh \left(m_{2}(\tau-p)\right)+\cosh \left(m_{2}(\tau-p)\right)\right]\right\} d p \\
& \quad+2 \mathrm{Gr} \sqrt{\frac{1+\mathrm{Rd}}{\pi \operatorname{Pr}}} \int_{0}^{\tau} \sqrt{p} e^{-m_{1}(\tau-p)}\left[\frac{\left(1-\lambda m_{1}\right)}{\lambda m_{2}} \sinh \left(m_{2}(\tau-p)\right)+\cosh \left(m_{2}(\tau-p)\right)\right] d p+\frac{\operatorname{Gr}(1+\mathrm{Rd})}{2 k_{1} \sqrt{\pi} \operatorname{Pr}} \\
& \quad \cdot \int_{0}^{\tau} \int_{0}^{p} \int_{0}^{\infty}\left\{\frac{(p-s)}{u} \sqrt{\frac{b}{s}} e^{-\left(\left(k_{2} s+u\right) / k_{1}+m_{1}(\tau-p)\right)} I_{1}\left(\frac{2}{k_{1}} \sqrt{b u s}\right)\left[\frac{\left(1-\lambda m_{1}\right)}{\lambda m_{2}} \sinh \left(m_{2}(\tau-p)\right)+\cosh \left(m_{2}(\tau-p)\right)\right]\right\} d u d s d p,
\end{aligned}
$$


and Nusselt number and skin friction for isothermal are given by

$$
\begin{aligned}
\mathrm{Nu} & =\frac{1}{\sqrt{\pi \tau}} \sqrt{\frac{\mathrm{Pr}}{1+\mathrm{Rd}}}, \\
F(\tau) & =-\frac{\mathrm{Gr}(1+\mathrm{Rd})}{k_{1} \operatorname{Pr}} \int_{0}^{\tau}\left\{e^{-m_{1}(\tau-p)}\left[\frac{\left(1-\lambda m_{1}\right)}{\lambda m_{2}} \sinh \left(m_{2}(\tau-p)\right)+\cosh \left(m_{2}(\tau-p)\right)\right]\right\} d p+\frac{\mathrm{Gr}(1+\mathrm{Rd})}{2 k_{1} \operatorname{Pr}} \\
& \cdot \sqrt{\frac{b}{\pi}} \int_{0}^{\tau} \int_{0}^{p} \int_{0}^{\infty}\left\{\frac{1}{u \sqrt{s}} e^{-\left(\left(k_{2} s+u\right) / k_{1}+m_{1}(\tau-p)\right)} I_{1}\left(\frac{2}{k_{1}} \sqrt{b u s}\right)\left[\frac{\left(1-\lambda m_{1}\right)}{\lambda m_{2}} \sinh \left(m_{2}(\tau-p)\right)+\cosh \left(m_{2}(\tau-p)\right)\right]\right\} d u d s d p \\
& +\mathrm{Gr} \sqrt{\frac{1+\mathrm{Rd}}{\pi \operatorname{Pr}}} \int_{0}^{\tau} \frac{e^{-m_{1}(\tau-p)}}{\sqrt{p}}\left[\frac{\left(1-\lambda m_{1}\right)}{\lambda m_{2}} \sinh \left(m_{2}(\tau-p)\right)+\cosh \left(m_{2}(\tau-p)\right)\right] d p .
\end{aligned}
$$

\section{Limiting Cases}

In this section, we discuss our present results and provide exact solutions for two special cases by taking suitable parameters equal to zero.

6.1. Absence of MHD and Radiation Parameter. By making Hartmann number $\mathrm{Ha}=0$ and radiation parameter $\mathrm{Rd}=0$, the obtained temperature profile (34) of isothermal case implies

$$
\theta(\eta, \tau)=\operatorname{erfc}\left(\frac{\eta \sqrt{\mathrm{Pr}}}{2 \sqrt{\tau}}\right)
$$

which is quite in agreement with [28, equation (18)]. Furthermore, by putting $\mathrm{Ha}=0$ and $\mathrm{Rd}=0$ in (35), it reduces to

$$
\begin{aligned}
u(\eta, \tau)= & \frac{\operatorname{Gr}\left(1+\lambda_{1}\right)}{\operatorname{Pr} \lambda m_{1}} \int_{0}^{\tau} e^{-m_{1}(\tau-p)-\eta / \sqrt{k_{1}}} \sinh \left(m_{1}(\tau-p)\right) d p \\
& +\frac{\operatorname{Gr}\left(1+\lambda_{1}\right)}{2 \lambda m_{1} k_{1} \operatorname{Pr}} \sqrt{\frac{k_{2}}{\pi}} \int_{0}^{\tau} \int_{0}^{p} \int_{0}^{\infty}\left\{\frac{\eta}{u \sqrt{s}} e^{-\left(m_{1}(\tau-p)+\left(k_{2} s+u\right) / k_{1}+\eta^{2} / 4 u\right)} \sinh \left(m_{1}(\tau-p)\right) I_{1}\left(\frac{2}{k_{1}} \sqrt{b u s}\right)\right\} d u d s d p \\
& -\frac{\operatorname{Gr}\left(1+\lambda_{1}\right)}{\operatorname{Pr} \lambda m_{1}} \int_{0}^{\tau} e^{-m_{1}(\tau-p)-\eta / \sqrt{k_{1}}} \sinh \left(m_{1}(\tau-p)\right) \operatorname{erfc}\left(\frac{\eta \sqrt{\operatorname{Pr}}}{2 \sqrt{p}}\right) d p
\end{aligned}
$$

which is identical to the solution obtained by Khan [28, equation (31)]. In Figure 2, it is observed that the graph of (40) matches well (31) in [28]. Hence, this fact confirms the accuracy of our results.

6.2. The Case of $\lambda_{1}=0$ (Second-Grade Fluid with MHD and Radiation Effects). Interestingly, by putting $\lambda_{1}=0$, the solutions in (31) and (35) can be reduced to secondgrade fluid with the effects of radiation and MHD. Thus, the velocity distribution for ramped wall temperature in (31) can be written as

$$
u(\eta, \tau)=u_{R 1}(\eta, \tau)-u_{R 1}(\eta, \tau-1) H(\tau-1),
$$

where

$$
\begin{gathered}
u_{R 1}(\eta, \tau)=\frac{\operatorname{Gr}(1+\mathrm{Rd})}{\operatorname{Pr} \lambda m_{2}} \int_{0}^{\tau} p e^{-\left(m_{1}(\tau-p)+\eta / \sqrt{k_{1}}\right)} \sinh \left(m_{2}(\tau-p)\right) d p+\frac{\operatorname{Gr}(1+\mathrm{Rd})}{2 \lambda k_{1} m_{2} \operatorname{Pr}} \\
\cdot \sqrt{\frac{b}{\pi}} \int_{0}^{\tau} \int_{0}^{p} \int_{0}^{\infty}\left\{\frac{\eta(p-s)}{u \sqrt{s}} e^{-\left(m_{1}(\tau-p)+\left(k_{2} s+u\right) / k_{1}+\eta^{2} / 4 u\right)} \sinh \left(m_{2}(\tau-p)\right) I_{1}\left(\frac{2}{k_{1}} \sqrt{b u s}\right)\right\} d u d s d p
\end{gathered}
$$




$$
\begin{aligned}
& -\frac{\mathrm{Gr}(1+\mathrm{Rd})}{\operatorname{Pr} \lambda m_{2}} \int_{0}^{\tau}\left\{e^{-m_{1}(\tau-p)}\left(p+\frac{\eta^{2}}{2} \frac{\mathrm{Pr}}{1+\mathrm{Rd}}\right) \sinh \left(m_{2}(\tau-p)\right) \operatorname{erfc}\left(\frac{\eta}{2 \sqrt{p}} \sqrt{\frac{\mathrm{Pr}}{1+\mathrm{Rd}}}\right)\right\} d p+\frac{\mathrm{Gr}}{\lambda m_{2}} \\
& \cdot \sqrt{\frac{1+\mathrm{Rd}}{\pi \operatorname{Pr}}} \int_{0}^{\tau}\left\{\eta \sqrt{p} e^{-\left(m_{1}(\tau-p)+\left(\eta^{2} / 4 p\right)(\operatorname{Pr} /(1+\mathrm{Rd}))\right)} \sinh \left(m_{2}(\tau-p)\right)\right\} d p
\end{aligned}
$$

and the velocity profile for isothermal case (35) reduces to

$$
\begin{aligned}
u(\eta, \tau)= & \frac{\operatorname{Gr}(1+\mathrm{Rd})}{\operatorname{Pr} \lambda m_{2}} \int_{0}^{\tau} e^{-m_{1}(\tau-p)-\eta / \sqrt{k_{1}}} \sinh \left(m_{2}(\tau-p)\right) d p \\
& +\frac{\mathrm{Gr}(1+\mathrm{Rd})}{2 k_{1} \operatorname{Pr} \lambda m_{2}} \sqrt{\frac{b}{\pi}} \int_{0}^{\tau} \int_{0}^{p} \int_{0}^{\infty}\left\{\frac{\eta}{u \sqrt{s}} e^{-\left(m_{1}(\tau-p)+\left(k_{2} s+u\right) / k_{1}+\eta^{2} / 4 u\right)} \sinh \left(m_{2}(\tau-p)\right) I_{1}\left(\frac{2}{k_{1}} \sqrt{b u s}\right)\right\} d u d s d p \\
& -\frac{\mathrm{Gr}(1+\mathrm{Rd})}{\operatorname{Pr} \lambda m_{2}} \int_{0}^{\tau} e^{-m_{1}(\tau-p)-\eta / \sqrt{k_{1}}} \sinh \left(m_{2}(\tau-p)\right) \operatorname{erfc}\left(\frac{\eta}{2 \sqrt{p}} \sqrt{\frac{\operatorname{Pr}}{1+\mathrm{Rd}}}\right) d p
\end{aligned}
$$

However, taking $\mathrm{Ha}=0, \mathrm{Rd}=0$, and $\mathrm{Gr}=1$ into (41) and (43) gives the well-known results

$$
\begin{aligned}
& u_{R 1}(\eta, \tau) \\
& =\frac{1}{\operatorname{Pr} \lambda m_{2}} \int_{0}^{\tau} p e^{-\left(m_{1}(\tau-p)+\eta / \sqrt{k_{1}}\right)} \sinh \left(m_{2}(\tau-p)\right) d p \\
& +\frac{1}{2 \lambda k_{1} m_{2} \operatorname{Pr}} \sqrt{\frac{b}{\pi}} \int_{0}^{\tau} \int_{0}^{p} \int_{0}^{\infty}\left\{\frac{\eta(p-s)}{u \sqrt{s}} e^{-\left(m_{1}(\tau-p)+\left(k_{2} s+u\right) / k_{1}+\eta^{2} / 4 u\right)} \sinh \left(m_{2}(\tau-p)\right) I_{1}\left(\frac{2}{k_{1}} \sqrt{b u s}\right)\right\} d u d s d p \\
& -\frac{1}{\operatorname{Pr} \lambda m_{2}} \int_{0}^{\tau}\left\{e^{-m_{1}(\tau-p)}\left(p+\frac{\eta^{2} \operatorname{Pr}}{2}\right) \sinh \left(m_{2}(\tau-p)\right) \operatorname{erfc}\left(\frac{\eta}{2} \sqrt{\frac{\operatorname{Pr}}{p}}\right)\right\} d p \\
& +\frac{1}{\lambda m_{2} \sqrt{\pi \operatorname{Pr}}} \int_{0}^{\tau}\left\{\eta \sqrt{p} e^{-\left(m_{1}(\tau-p)+\eta^{2} \operatorname{Pr} / 4 p\right)} \sinh \left(m_{2}(\tau-p)\right)\right\} d p \\
& u(\eta, \tau) \\
& =\frac{1}{\operatorname{Pr} \lambda m_{2}} \int_{0}^{\tau} e^{-m_{1}(\tau-p)-\eta / \sqrt{k_{1}}} \sinh \left(m_{2}(\tau-p)\right) d p \\
& +\frac{1}{2 k_{1} \operatorname{Pr} \lambda m_{2}} \sqrt{\frac{b}{\pi}} \int_{0}^{\tau} \int_{0}^{p} \int_{0}^{\infty}\left\{\frac{\eta}{u \sqrt{s}} e^{-\left(m_{1}(\tau-p)+\left(k_{2} s+u\right) / k_{1}+\eta^{2} / 4 u\right)} \sinh \left(m_{2}(\tau-p)\right) I_{1}\left(\frac{2}{k_{1}} \sqrt{b u s}\right)\right\} d u d s d p \\
& -\frac{1}{\operatorname{Pr} \lambda m_{2}} \int_{0}^{\tau} e^{-m_{1}(\tau-p)-\eta / \sqrt{k_{1}}} \sinh \left(m_{2}(\tau-p)\right) \operatorname{erfc}\left(\frac{\eta}{2} \sqrt{\frac{\operatorname{Pr}}{p}}\right) d p .
\end{aligned}
$$




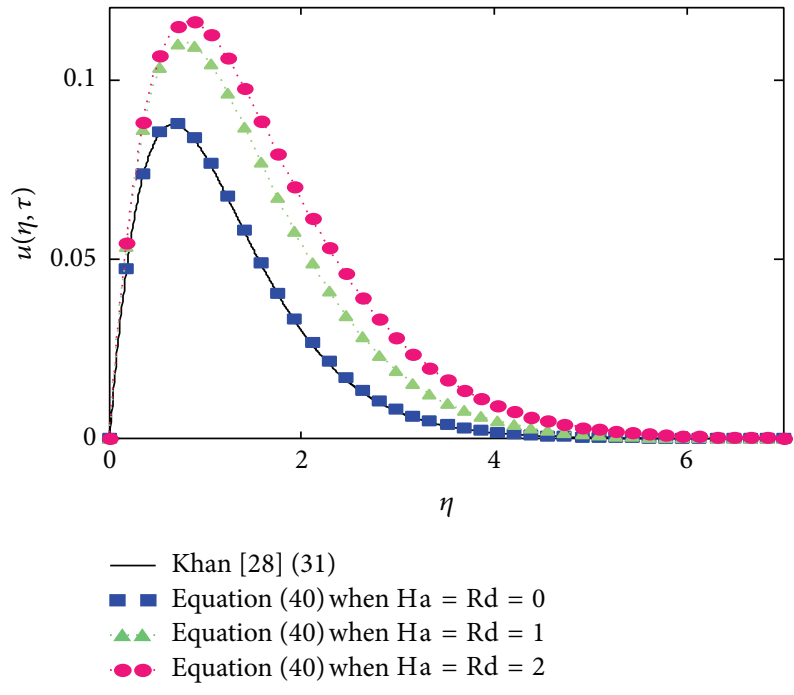

FIGURE 2: Comparison of velocity $u(\eta, \tau)$ in (40) with (31) obtained by Khan [28].

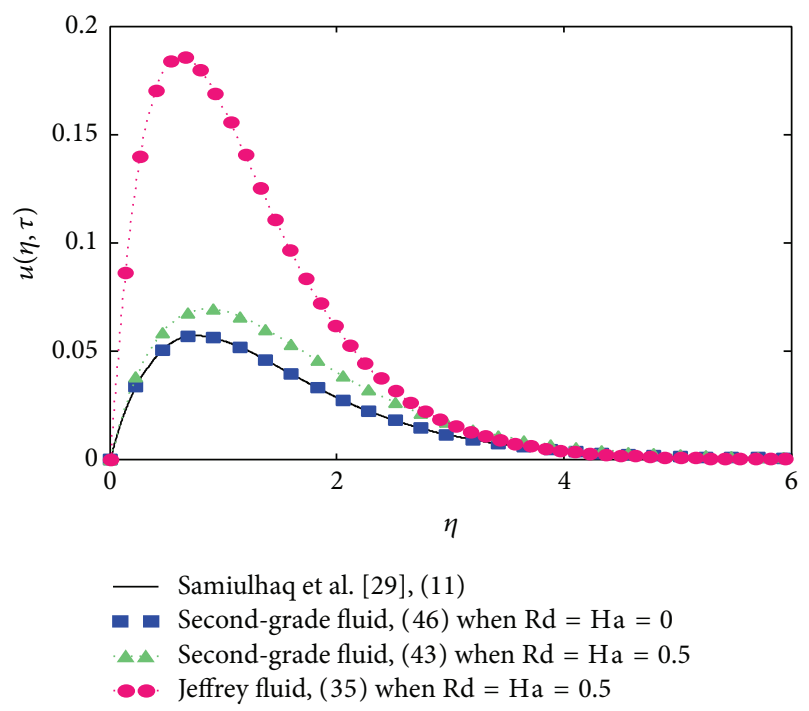

Figure 3: Comparison of velocity $u(\eta, \tau)$ for isothermal in (46) with (11) obtained by Samiulhaq et al. [29].

Equation (46) is identical to the published results obtained by Samiulhaq et al. [29, equation (11)]. Furthermore, in Figure 3 it is showed that the graph of (46) matches well (11) in [29]. Hence, we can say the results are found to be in excellent agreement.

\section{Results and Discussions}

Numerical computation of analytical expressions (20), (31), (34), and (35) of the present problem has been carried out for various values of embedded parameters, namely, dimensionless Jeffrey fluid parameter $\lambda$, Prandtl number Pr, Grashof number Gr, Hartmann number Ha, radiation parameter Rd, and dimensionless time $\tau$. The velocity and

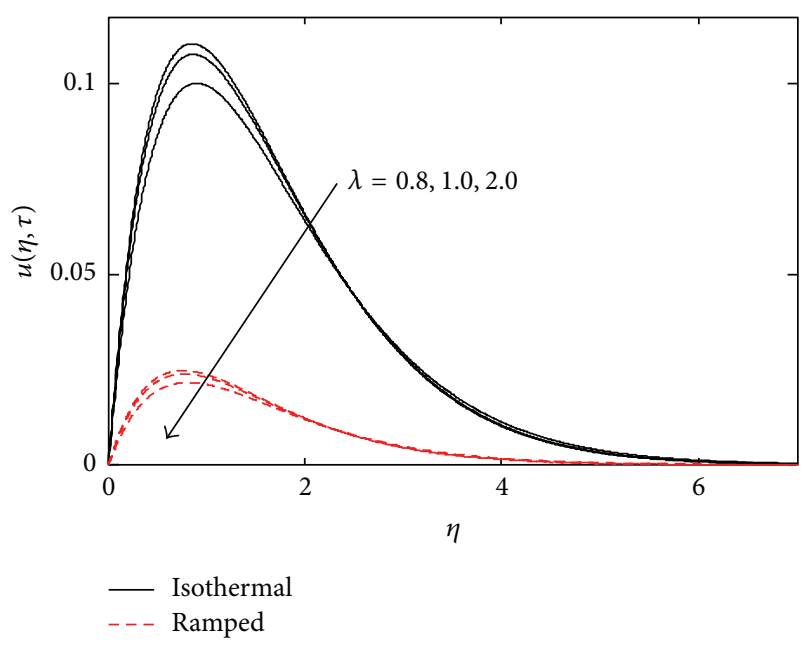

FIGURE 4: Velocity profile for different values of $\lambda$, when $\operatorname{Pr}=0.71$, $\mathrm{Rd}=2, \mathrm{Gr}=1, \mathrm{Ha}=2$, and $\tau=0.5$.

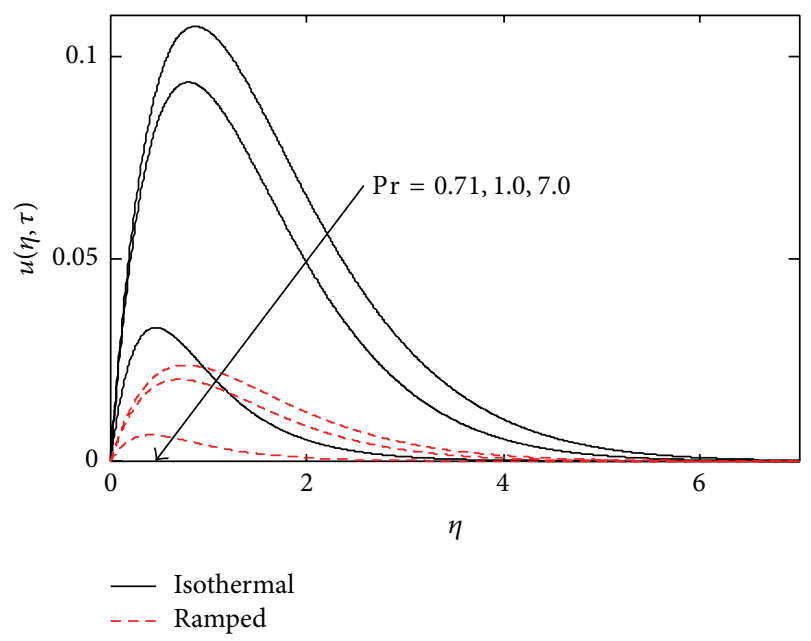

FIGURE 5: Velocity profile for different values of $\operatorname{Pr}$, when $\lambda=1$, $\mathrm{Rd}=2, \mathrm{Gr}=1, \mathrm{Ha}=2$, and $\tau=0.5$.

temperature profiles are illustrated graphically in Figures 412 in order to give clear insight of physical problem of Jeffrey fluid.

The influence of Jeffrey fluid parameter $\lambda$ on velocity profile is shown in Figure 4. It is found that the momentum boundary layer thickness increases as the Jeffrey fluid parameter effect is strengthened. Hence, the velocity profile decreases due to this fact. Moreover, the fluid velocity for ramped wall temperature is always lower compared to the case of isothermal. The variations of velocity profile for three different values of Prandtl number $\operatorname{Pr}=0.71,1.0,7.0$, which correspond to air, electrolyte solution, and water, respectively, are plotted in Figure 5. It is noticed that the increase of $\mathrm{Pr}$ makes the fluid motion slower. Physically, it is justified due to the fact that the fluid with high Prandtl number has high viscosity which makes the fluid thick. As a result the velocity decreases. 


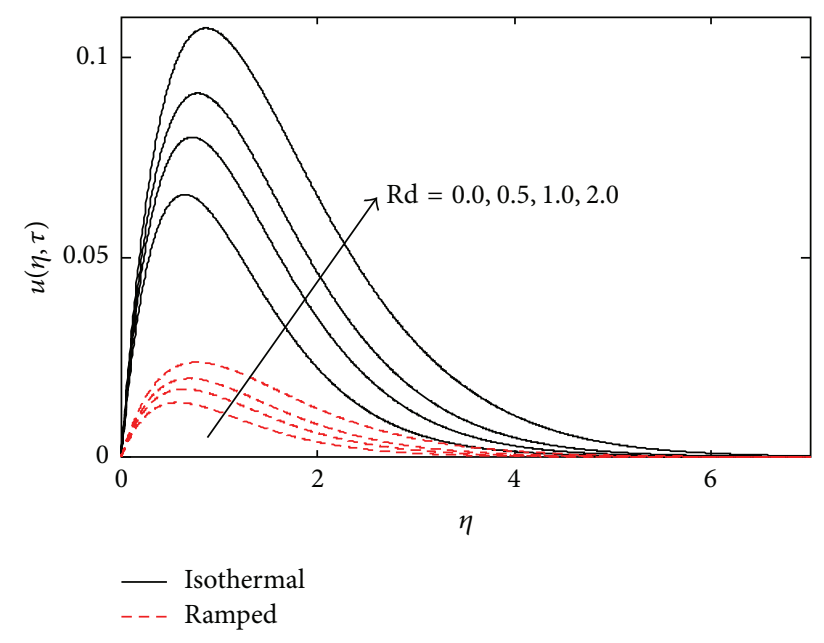

FIGURE 6: Velocity profile for different values of $\mathrm{Rd}$, when $\lambda=1$, $\operatorname{Pr}=0.71, \mathrm{Gr}=1, \mathrm{Ha}=2$, and $\tau=0.5$.

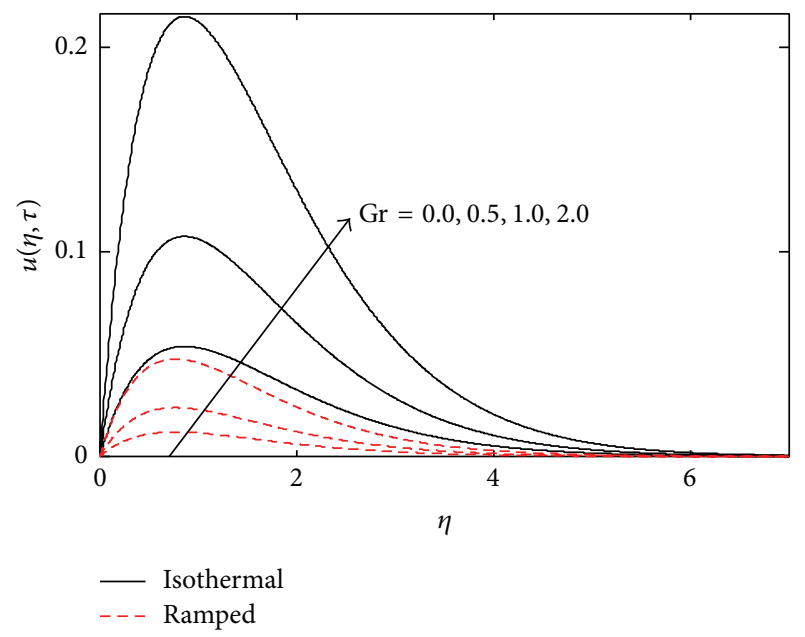

FIGURE 7: Velocity profile for different values of $\mathrm{Gr}$, when $\lambda=1$, $\operatorname{Pr}=0.71, \mathrm{Rd}=2, \mathrm{Ha}=2$, and $\tau=0.5$.

Further, the effect of radiation parameter $\mathrm{Rd}$ on the velocity profile is depicted in Figure 6. The trend shows that velocity increases with increasing values of radiation parameter. This is because when the intensity of radiation parameter increased which in turn increased the rate of energy transport to the fluids, the bond holding the components of the fluid particles is easily broken and thus decreases the viscosity. This will make the fluid move faster which leads to increase of the velocity profile. The graphical results for Grashof number are presented in Figure 7. Physically, the Grashof number is defined as the ratio of buoyancy forces to viscous forces. Large values of $\mathrm{Gr}$ enhance the buoyancy forces which gives rise to an increase in the induced flow.

The effect of Hartmann number Ha upon velocity $u(\eta, \tau)$ is elucidated from Figure 8. As expected, an increase in $\mathrm{Ha}$ reduces the velocity profile. This is because of the Lorentz force which arises due to the application of magnetic field to an electrically conducting fluid and gives rise to a resistance

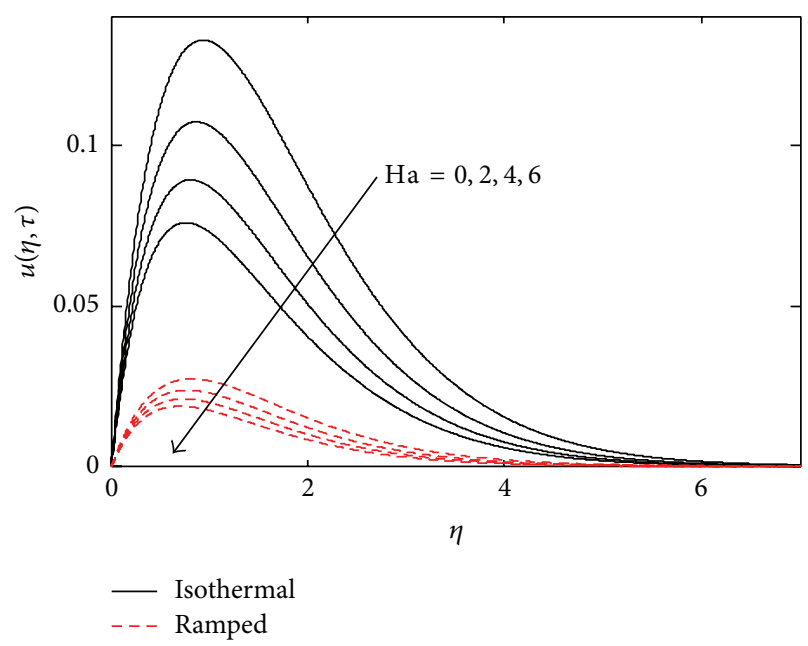

FIGURE 8: Velocity profile for different values of Ha, when $\lambda=1$, $\operatorname{Pr}=0.71, \operatorname{Rd}=2, \mathrm{Gr}=1$, and $\tau=0.5$.

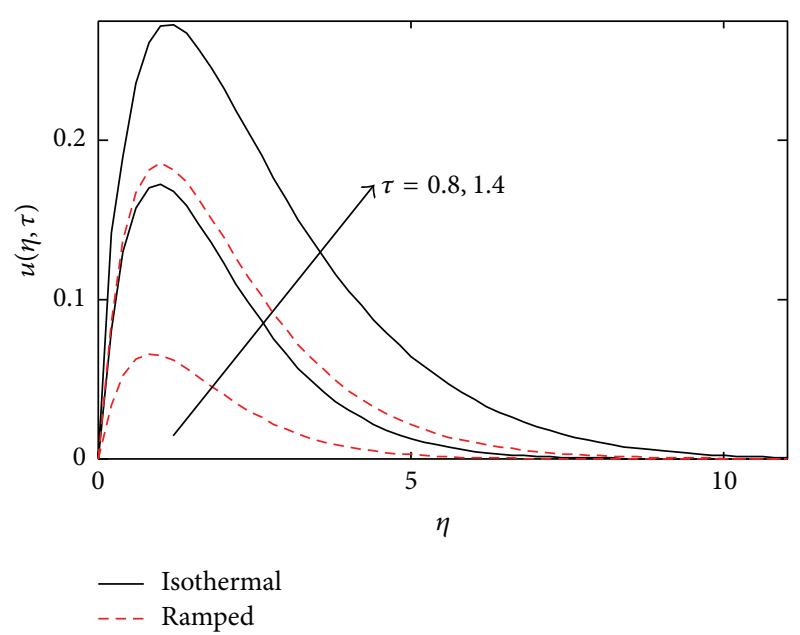

FIGURE 9: Velocity profile for different values of $\tau$, when $\lambda=1, \operatorname{Pr}=$ $0.71, \mathrm{Rd}=2, \mathrm{Gr}=1$, and $\mathrm{Ha}=2$.

force. Due to this force, the motion of fluid flow in momentum boundary layer tends to retard. On the other hand, Figure 9 shows the effect of dimensionless time $\tau$ towards velocity $u(\eta, \tau)$. It can be seen from the figure that velocity is an increasing function of $\tau$.

Figure 10 reveals that the temperature profile is reduced when $\mathrm{Pr}$ is increased. The explanation for such behavior lies in the fact that Prandtl number is defined as the ratio of momentum diffusivity to thermal diffusivity. Therefore, at smaller values of Pr fluids possess high thermal conductivity which makes the heat diffuse away from the heated surface more rapidly and faster compared to higher values of Prandtl number. Thus, increase the boundary layer thickness and consequently decrease the temperature distribution. The influence of radiation $\mathrm{Rd}$ on temperature field is demonstrated in Figure 11. As anticipated, an increase in $\mathrm{Rd}$ increases the temperature profile since radiation parameter signifies the relative contribution of conduction heat transfer to thermal 


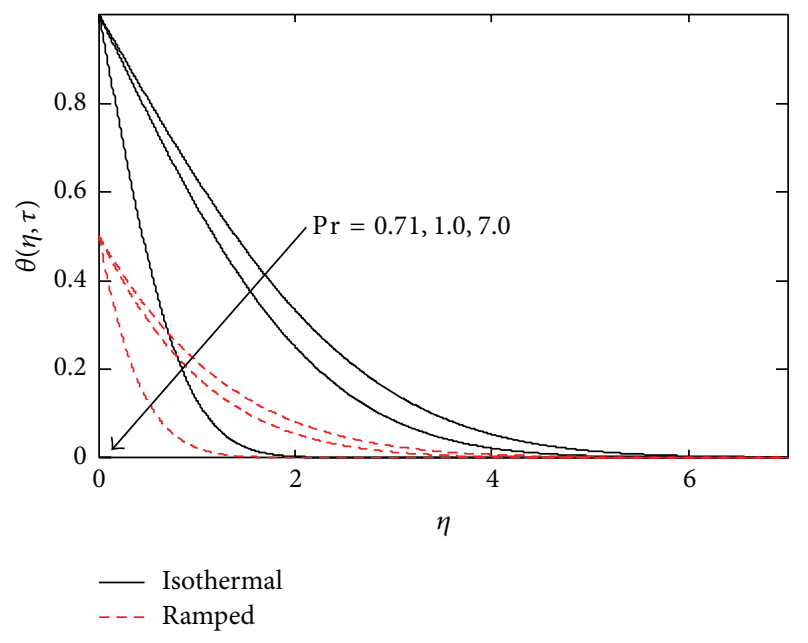

FIGURE 10: Temperature profile for different values of Pr, when Rd = 2 and $\tau=0.5$.

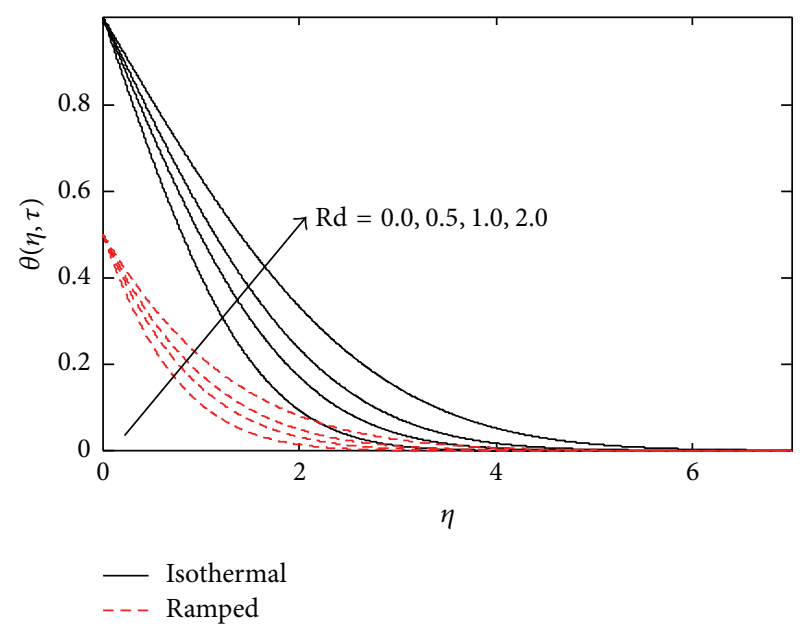

FIGURE 11: Temperature profile for different values of $\mathrm{Rd}$, when $\mathrm{Pr}=$ 0.71 and $\tau=0.5$.

radiation transfer. Finally, the effect of dimensionless time $\tau$ on temperature profiles is sketched in Figure 12. It is also clear that temperature is an increasing function of $\tau$.

\section{Conclusion}

This study presents a theoretical investigation of thermal radiation in unsteady MHD free convection flow of Jeffrey fluid with ramped wall temperature. The dimensionless governing equations are solved by using the Laplace transform method. Graphical results for velocity and temperature are obtained to understand the physical behavior for several embedded parameters. The results show that increasing $\lambda$, $\mathrm{Rd}$, and $\tau$ decreases fluid velocity for both ramped wall temperature and isothermal plates. Meanwhile, the behavior of velocity and temperature profiles for air is greater than water. However, increasing Gr boosts the fluid motion due to the enhancement in buoyancy force. As expected, the effect of Ha shows the opposite behavior. It is because the Lorentz

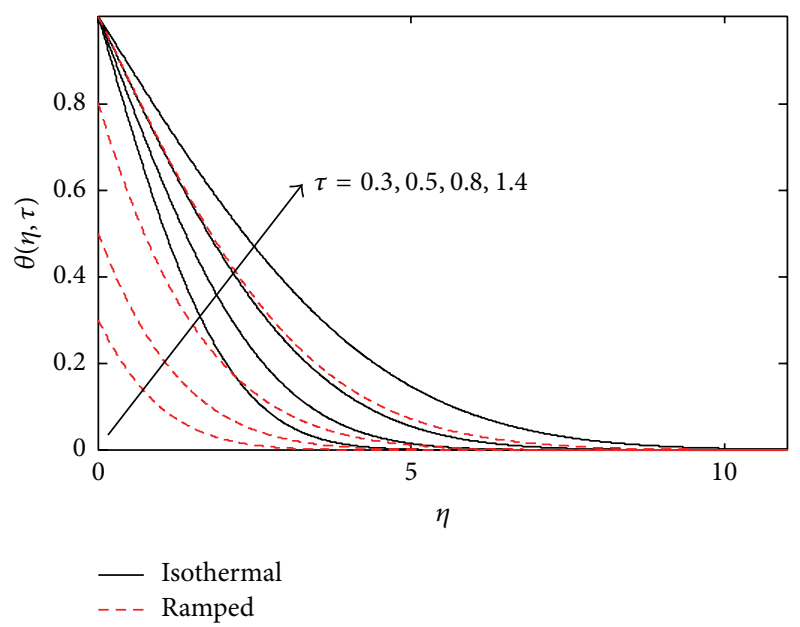

FIGURE 12: Temperature profile for different values of $\tau$, when $\operatorname{Pr}=$ 0.71 and $\mathrm{Rd}=2$.

force tends to decelerate the fluid flow. Further, the influences of $\mathrm{Rd}$ and $\tau$ increase the temperature profile. Finally, we observed that the boundary layer thickness for ramped wall temperature is always less than isothermal plate.

\section{Conflict of Interests}

The authors of this paper do not have any conflict of interests regarding its publication.

\section{References}

[1] S. Nadeem, B. Tahir, F. Labropulu, and N. S. Akbar, "Unsteady oscillatory stagnation point flow of a Jeffrey fluid," Journal of Aerospace Engineering, vol. 27, no. 3, pp. 636-643, 2014.

[2] T. Hayat and M. Mustafa, "Influence of thermal radiation on the unsteady mixed convection flow of a Jeffrey fluid over a stretching sheet," Zeitschrift für Naturforschung, vol. 65, pp. 711719,2010

[3] T. Hayat, S. A. Shehzad, M. Qasim, and S. Obaidat, "Radiative flow of Jeffery fluid in a porous medium with power law heat flux and heat source," Nuclear Engineering and Design, vol. 243, pp. 15-19, 2012.

[4] S. A. Shehzad, A. Alsaedi, and T. Hayat, "Influence of thermophoresis and joule heating on the radiative flow of Jeffrey fluid with mixed convection," Brazilian Journal of Chemical Engineering, vol. 30, no. 4, pp. 897-908, 2013.

[5] S. A. Shehzad, Z. Abdullah, A. Alsaedi, F. M. Abbasi, and T. Hayat, "Thermally radiative three-dimensional flow of Jeffrey nanofluid with internal heat generation and magnetic field," Journal of Magnetism and Magnetic Materials, vol. 397, pp. 108114, 2016.

[6] T. Hussain, S. A. Shehzad, T. Hayat, A. Alsaedi, F. Al-Solamy, and M. Ramzan, "Radiative hydromagnetic flow of Jeffrey nanofluid by an exponentially stretching sheet," PLOS ONE, vol. 9, no. 8, Article ID e103719, 9 pages, 2014.

[7] K. Das, N. Acharya, and P. K. Kundu, "Radiative flow of MHD Jeffrey fluid past a stretching sheet with surface slip and melting heat transfer," Alexandria Engineering Journal, vol. 54, no. 4, pp. 815-821, 2015. 
[8] T. Hayat and N. Ali, "Peristaltic motion of a Jeffrey fluid under the effect of a magnetic field in a tube," Communications in Nonlinear Science and Numerical Simulation, vol. 13, no. 7, pp. 1343-1352, 2008.

[9] A. M. Abd-Alla, S. M. Abo-Dahab, and M. M. Albalawi, "Magnetic field and gravity effects on peristaltic transport of a Jeffrey fluid in an asymmetric channel," Abstract and Applied Analysis, vol. 2014, Article ID 896121, 11 pages, 2014.

[10] A. M. Abd-Alla and S. M. Abo-Dahab, "Magnetic field and rotation effects on peristaltic transport of a Jeffrey fluid in an asymmetric channel," Journal of Magnetism and Magnetic Materials, vol. 374, pp. 680-689, 2015.

[11] S. Akram and S. Nadeem, "Influence of induced magnetic field and heat transfer on the peristaltic motion of a Jeffrey fluid in an asymmetric channel: closed form solutions," Journal of Magnetism and Magnetic Materials, vol. 328, pp. 11-20, 2013.

[12] K. Kavita, K. Ramakrishna Prasad, and B. Aruna Kumari, "Influence of heat transfer on MHD oscillatory flow of jeffrey fluid in a channel," Advances in Applied Science Research, vol. 3, no. 4, pp. 2312-2325, 2012.

[13] A. S. Idowu, K. M. Joseph, and S. Daniel, "Effect of heat and mass transfer on unsteady MHD oscillatory flow of Jeffrey fluid in a horizontal channel with chemical reaction," IOSR Journal of Mathematics, vol. 8, no. 5, pp. 74-87, 2013.

[14] A. Ali and S. Asghar, "Analytic solution for oscillatory flow in a channel for Jeffrey fluid," Journal of Aerospace Engineering, vol. 27, no. 3, pp. 644-651, 2014.

[15] M. Khan, "Partial slip effects on the oscillatory flows of a fractional Jeffrey fluid in a porous medium," Journal of Porous Media, vol. 10, no. 5, pp. 473-487, 2007.

[16] T. Hayat, M. Khan, K. Fakhar, and N. Amin, "Oscillatory rotating flows of a fractional Jeffrey fluid filling a porous space," Journal of Porous Media, vol. 13, no. 1, pp. 29-38, 2010.

[17] S. Nadeem, A. Hussain, and M. Khan, "Stagnation flow of a Jeffrey fluid over a shrinking sheet," Zeitschrift für Naturforschung, vol. 65, no. 6-7, pp. 540-548, 2010.

[18] M. A. A. Hamad, S. M. AbdEl-Gaied, and W. A. Khan, "Thermal jump effects on boundary layer flow of a jeffrey fluid near the stagnation point on a stretching/shrinking sheet with variable thermal conductivity," Journal of Fluids, vol. 2013, Article ID 749271, 8 pages, 2013.

[19] T. Hayat, S. Asad, M. Mustafa, and A. Alsaedi, "MHD stagnation-point flow of Jeffrey fluid over a convectively heated stretching sheet," Computers \& Fluids, vol. 108, pp. 179-185, 2015.

[20] M. Qasim, "Heat and mass transfer in a Jeffrey fluid over a stretching sheet with heat source/sink," Alexandria Engineering Journal, vol. 52, no. 4, pp. 571-575, 2013.

[21] N. Dalir, "Numerical study of entropy generation for forced convection flow and heat transfer of a Jeffrey fluid over a stretching sheet," Alexandria Engineering Journal, vol. 53, no. 4, pp. 769-778, 2014.

[22] N. Dalir, M. Dehsara, and S. S. Nourazar, "Entropy analysis for magnetohydrodynamic flow and heat transfer of a Jeffrey nanofluid over a stretching sheet," Energy, vol. 79, pp. 351-362, 2015.

[23] T. Hayat, M. Awais, and S. Obaidat, "Three-dimensional flow of a Jeffery fluid over a linearly stretching sheet," Communications in Nonlinear Science and Numerical Simulation, vol. 17, no. 2, pp. 699-707, 2012.
[24] T. Hayat, M. Awais, and A. Alsaedi, "Newtonian heating and magnetohydrodynamic effects in flow of a Jeffery fluid over a radially stretching surface," International Journal of the Physical Sciences, vol. 7, no. 21, pp. 2838-2844, 2012.

[25] T. Hayat, S. Asad, M. Qasim, and A. A. Hendi, "Boundary layer flow of a Jeffrey fluid with convective boundary conditions," International Journal for Numerical Methods in Fluids, vol. 69, no. 8, pp. 1350-1362, 2012.

[26] S. A. Shehzad, T. Hayat, M. S. Alhuthali, and S. Asghar, "MHD three-dimensional flow of Jeffrey fluid with newtonian heating," Journal of Central South University, vol. 21, no. 4, pp. 1428-1433, 2014.

[27] M. Farooq, N. Gull, A. Alsaedi, and T. Hayat, "MHD flow of a Jeffrey fluid with Newtonian heating," Journal of Mechanics, vol. 31, no. 3, pp. 319-329, 2015.

[28] I. Khan, "A note on exact solutions for the unsteady free convection flow of a jeffrey fluid," Zeitschrift für Naturforschung A, vol. 70, no. 6, pp. 272-284, 2015.

[29] Samiulhaq, I. Khan, F. Ali, and S. Shafie, "Free convection flow of a second-grade fluid with ramped wall temperature," Heat Transfer Research, vol. 45, no. 7, pp. 579-588, 2014. 


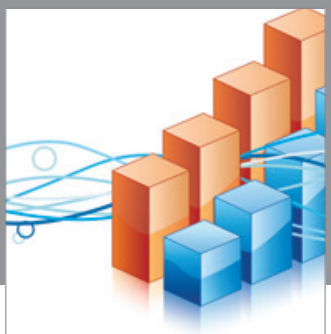

Advances in

Operations Research

vatem alat4

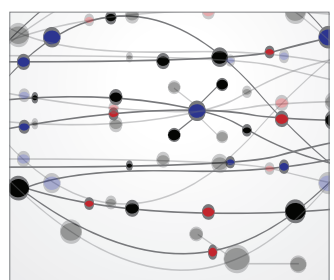

\section{The Scientific} World Journal
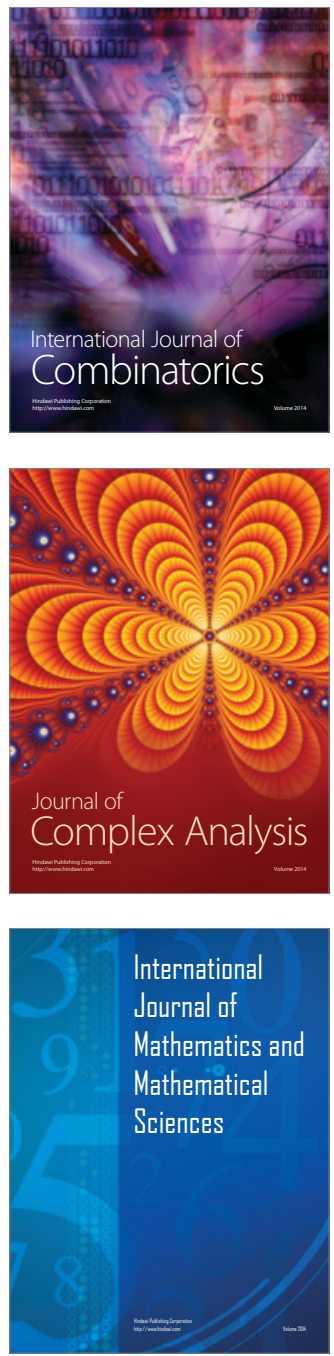
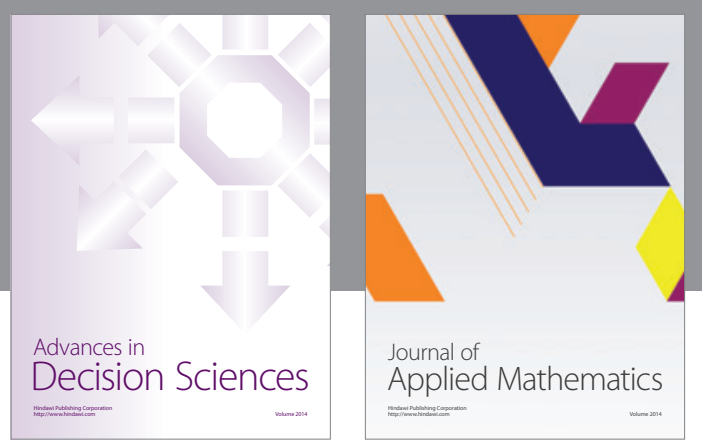

Algebra

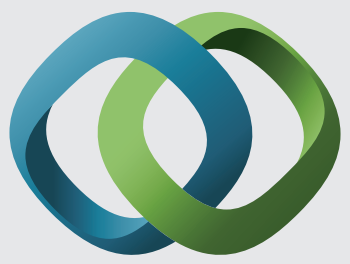

\section{Hindawi}

Submit your manuscripts at

http://www.hindawi.com
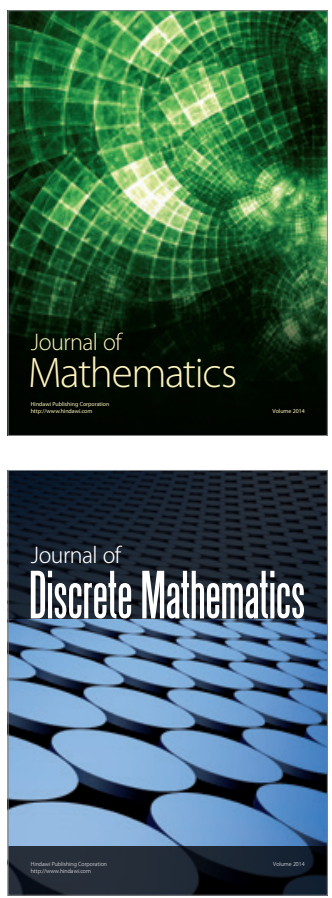

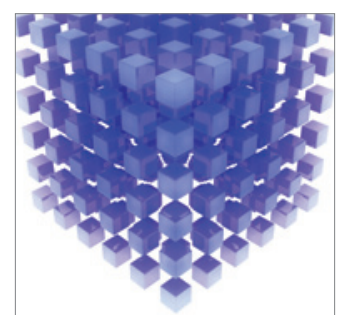

Mathematical Problems in Engineering
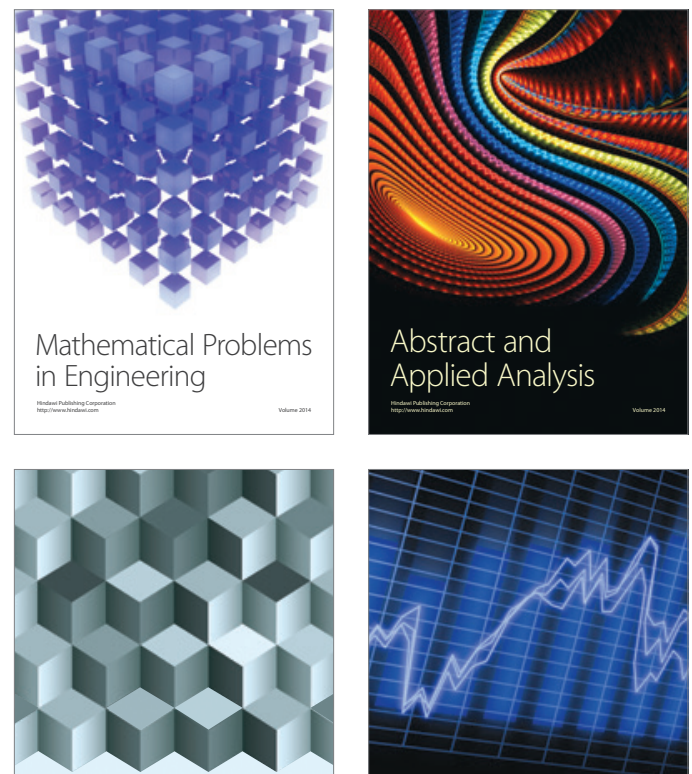

Journal of

Function Spaces

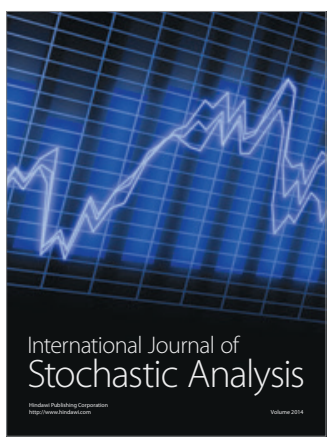

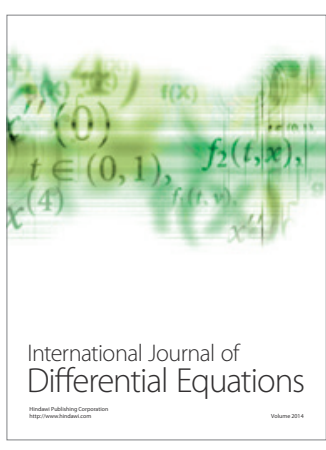
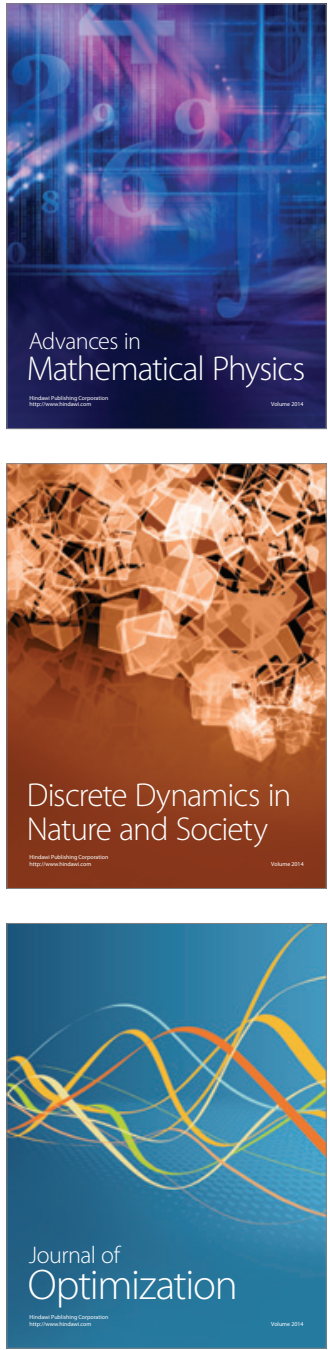